\title{
Genotype-phenotype correlations of adult- onset PLA2G6-associated Neurodegeneration: case series and literature review
}

\author{
Yung-Tsai Chu', Han-Yi Lin ${ }^{1}$, Pei-Lung Chen ${ }^{2,3}$ and Chin-Hsien Lin ${ }^{1 *}$
}

\begin{abstract}
Background: Phospholipase A2 group VI (PLA2G6) mutations associated with neurodegeneration (PLAN) manifest as heterogeneous neurodegenerative disorders with variable ages of onset. The genotype-phenotype correlation is not well-established. We aim to describe three adult patients with PLAN and combined these data with results from previous studies to elucidate adult-onset PLA2G6 phenotype-genotype correlations.

Case presentations: The first index patient presented with dystonia-parkinsonism starting at age 31 years, accompanied by major depression and cognitive decline. Genetic analysis using targeted next generation sequencing (NGS) panel, Sanger sequencing, and segregation analyses revealed a compound heterozygous mutation, c.991G > T (p.D331Y)/c.1077G > A (M358lfSX), in PLA2G6. The other two patients had levodopa-responsive, early-onset parkinsonism, starting in their late twenties. Both patients had homozygous c.991G > T (p.D331Y) mutations in PLA2G6. Patient characteristics of our reported 3 cases were compared to those of 32 previously described (2008 to 2019) patients with adult-onset PLAN. Among the combined cohort of 35 patients with adult-onset PLAN, 14 had dystonia-parkinsonism, 17 had early-onset Parkinson's disease, 3 had hereditary spastic paraparesis, and one had ataxia. The c.991G > T (p. D331Y) mutation was almost exclusively found in Chinese patients, suggesting a common founder effect. All patients with homozygous p.D331Y mutations had levodopa-responsive, early-onset PD (100\%); while other mutations mostly led to dystonia-parkinsonism, ataxia, spasticity, and combine psychiatric comorbidities.
\end{abstract}

Conclusions: We showed that adult-onset PLAN could present as purely parkinsonism features, without brain iron accumulation, particularly patients with homozygous p.D331Y mutations. Compound heterozygous mutations, including heterozygous p.D331Y, produced heterogeneous phenotypes, without obvious levodopa responsiveness.

Keywords: PLA2G6, Dystonia-parkinsonism, Early-onset parkinsonism, Hereditary spastic paraparesis, Ataxia, PLA2G6associated neurodegeneration

\section{Background}

PLA2G6-associated neurodegeneration (PLAN) is a heterogeneous group of neurodegenerative disorders that

\footnotetext{
*Correspondence: q93421022@ntu.edu.tw

'Department of Neurology, National Taiwan University Hospital, No. 7, Chung-Shan South Road, Taipei 100, Taiwan

Full list of author information is available at the end of the article
}

result from mutations in the phospholipase A2 group VI gene (PLA2G6) $[1,2]$. The PLA2G6 gene encodes a group of VIA calcium-independent phospholipase A2 proteins. Phospholipase A2 is an enzyme involved in phospholipid metabolism, and it is essential for maintaining cell membrane integrity $[1,2]$. PLAN can be classified into four subtypes, based on onset age, including: infantile neuroaxonal

C C The Author(s). 2020 Open Access This article is licensed under a Creative Commons Attribution 4.0 International License, which permits use, sharing, adaptation, distribution and reproduction in any medium or format, as long as you give appropriate credit to the original author(s) and the source, provide a link to the Creative Commons licence, and indicate if changes were made. The images or other third party material in this article are included in the article's Creative Commons licence, unless indicated otherwise in a credit line to the material. If material is not included in the article's Creative Commons licence and your intended use is not permitted by statutory regulation or exceeds the permitted use, you will need to obtain permission directly from the copyright holder. To view a copy of this licence, visit http://creativecommons.org/licenses/by/4.0/ The Creative Commons Public Domain Dedication waiver (http://creativecommons.org/publicdomain/zero/1.0/) applies to the data made available in this article, unless otherwise stated in a credit line to the data. 
dystrophy (INAD), atypical neuroaxonal dystrophy (ANAD), dystonia-parkinsonism, and autosomal recessive early-onset parkinsonism (known as PARK14) [3]. INAD and ANAD onsets occur in childhood. Brain magnetic resonance image (MRI) findings have revealed that most patients have cerebellar cortical atrophy and iron deposition in the globus pallidus and substantia nigra. These findings are known as neurodegeneration with brain iron accumulation, type II (NBIA II) [4]. In contrast to childhood-onset PLAN, adult-onset PLAN is associated with widely variable clinical manifestations, and the genotype-phenotype correlation has not been well established. Here, we describe three patients with adult-onset PLAN. We compared the clinical features, treatment responses, and radiological findings of these patients to those reported for 43 patients described in previous studies to elucidate the phenotypegenotype correlations in adult-onset PLAN.

\section{Case presentation}

Patient 1 was a 36 -year-old man, with normal birth and developmental milestones. He had a history of depression for 18 years but had not used anti-depressant or neuroleptic agents consistently. Several times, suicide was attempted. At age of 31 years, motor symptoms gradually developed. He had progressive onset of spastic dysarthria and hypophonia, predominantly left-sided bradykinesia, and dystonia in the left hand ensued. Three years after the onset of motor symptoms, he was confined to a wheelchair with anarthria, and he was totally dependent on assistance from others in daily living activities. In addition, he experienced several episodes of oculogyric crisis. He did not have a relevant family history (Fig. 1a).
He was then brought to our movement disorder clinic for evaluation at the age of 36 years. The neurological examination revealed oromandibular, truncal, and limb dystonia and generalized rigidity and bradykinesia; the part III motor score of the Unified Parkinson's Disease Rating Scale (UPDRS) was 60 out of 108. He scored 21 out of 30 on the Mini-Mental State Examination (MMSE). There were no Kayser-Fleischer rings during the physical examination. A brain MRI showed diffuse cortical and cerebellar atrophy, with no abnormal iron deposits in the basal ganglia (Fig. 2a-c). A Tc-99 m TRODAT single-photon emission computed tomography scan revealed markedly reduced dopamine transporter activity in the bilateral basal ganglia (Fig. 2d). ${ }^{18} \mathrm{~F}$ labeled fluoro-deoxyglucose positron emission tomography (FDG-PET) scan demonstrated hypometabolism in the bilateral parieto-occipital lobes. The patient was treated with levodopa (400 mg per day) and benserazide (50 $\mathrm{mg}$ three times per day), a rotigotine transdermal patch ( $8 \mathrm{mg}$ per day), and clonazepam $(0.5 \mathrm{mg})$. These medications improved motor function to the extent that he could walk with assistance, and the UPDRS part III motor score was 40 out of 108 after treatment.

The results of laboratory tests were within normal limits, including normal plasma ceruloplasmin levels and 24-h urine copper excretion. We then performed comprehensive genetic analyses for the index patient with a capture-based next generational sequencing targeted gene panel that covered more than 40 genes related to Parkinson's disease (PD) and neurodegenerative disorders using similar methods described previously [5]. Mutations were verified with Sanger sequencing and dye-
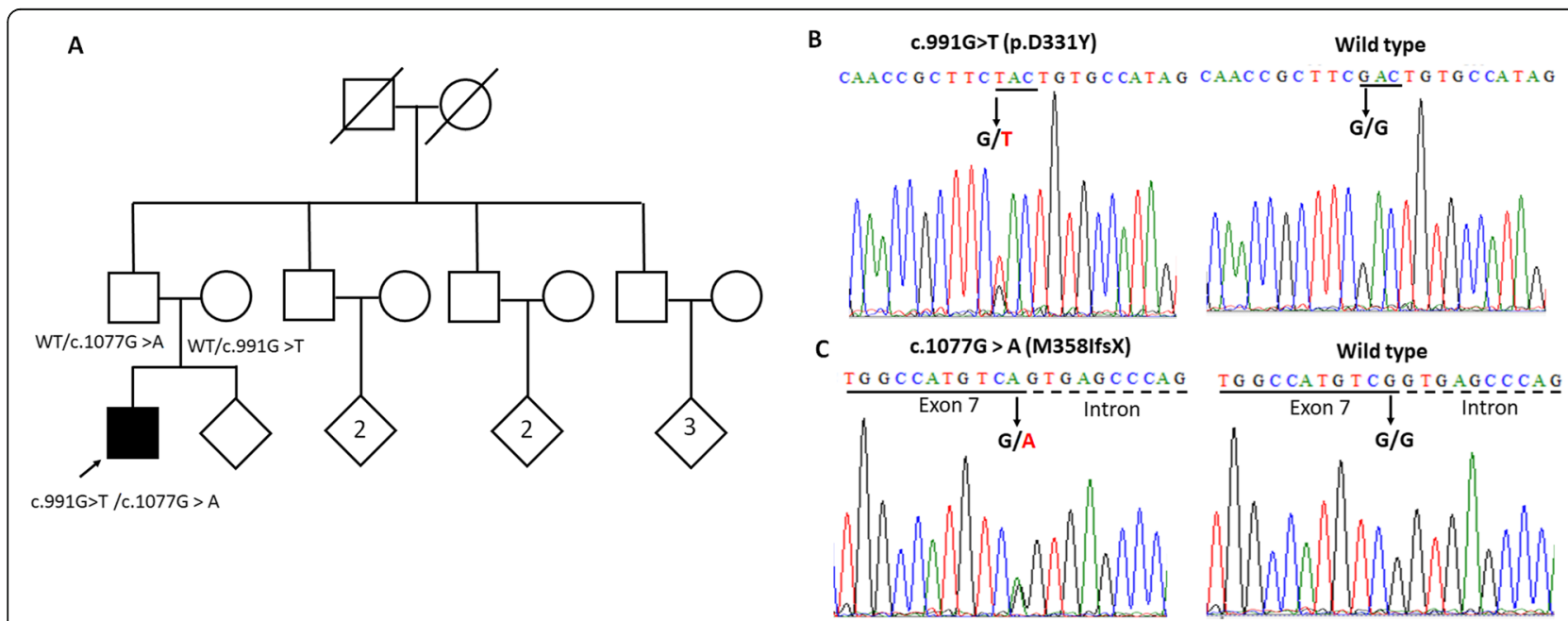

Fig. 1 Family pedigree and genetic analysis of an index family. (a) Pedigree of an index family with compound heterozygous c.991G > T (p.Asp331Tyr) and c.1077G > A (M358lfSX) mutations in PLA2G6. WT, wild type. Open symbol: unaffected; filled symbol: affected; symbol with a diagonal line: deceased; diamond: total number of children, unknown sex; arrow: proband. (b) Sanger sequencing traces confirm the heterozygous PLA2G6 c.991G > T (p.Asp331Tyr) missense mutation. (c) Sanger sequencing traces confirm the heterozygous PLA2G6 c.1077G > A (M358lfsX) frame-shift mutation 

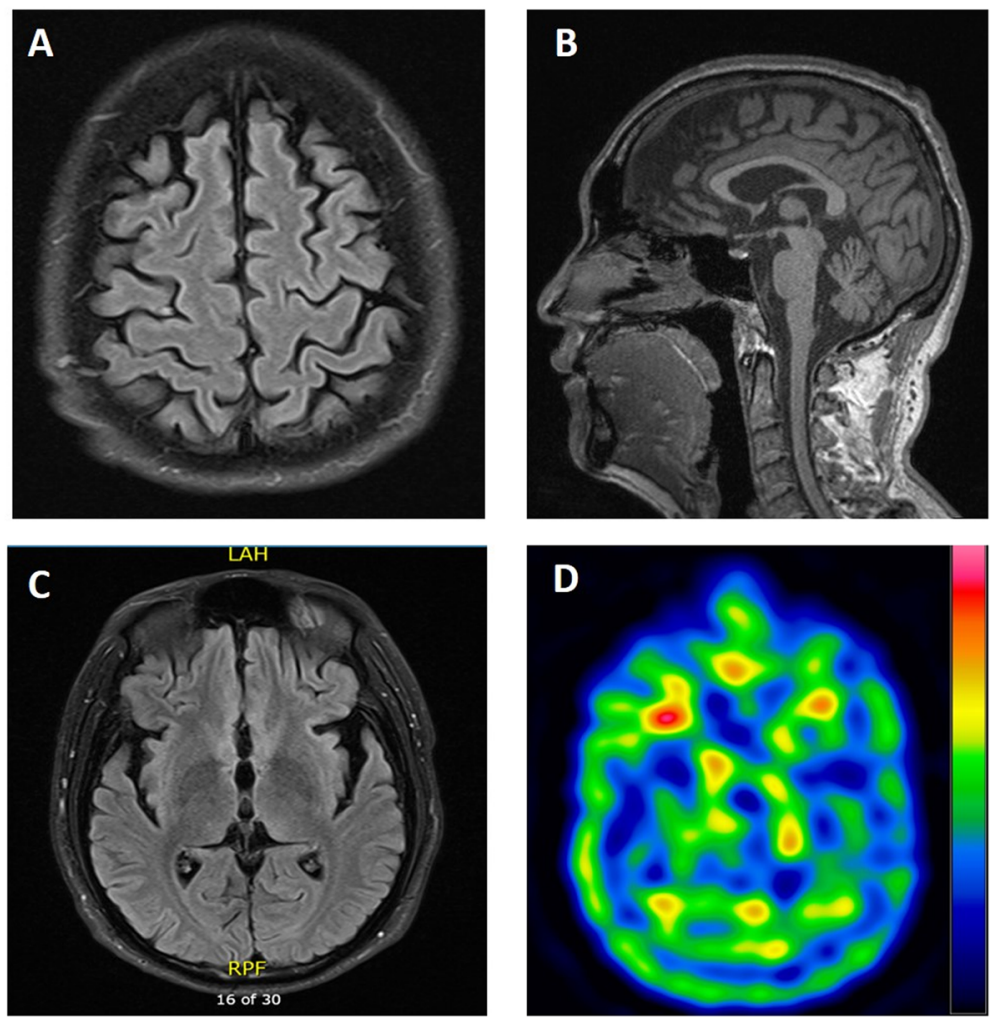

Fig. 2 Brain imaging findings in the first index patient. (a, b, c) Brain MRIs with T2 Fluid Attenuated inversion recovery (FLAIR) sequence show (a) diffuse atrophy in the cortex and cerebellum and (b) cerebellar atrophy, but (c) no abnormal signs of iron accumulation in the globus pallidus. (d) A Tc-99 m TRODAT scan reveals reduced dopamine transporter activity in the bilateral basal ganglia

terminator chemistry in an Automated Sequencer Genetic Analyzer (model 3100; Applied Biosystems, Foster City, CA, USA). We identified the pathogenic missense variant, c.991G $>$ T (p.D331Y), and a c.1077G > A (M358IfsX) frame-shift mutation in PLA2G6 (Fig. 1b,c). Direct Sanger sequencing verified the c.991G $>$ T (p.D331Y) missense mutation in both the proband and the asymptomatic mother (Fig. 2a,b). The c.1077G > A (M358IfsX) frame-shift mutation in PLA2G6 was confirmed with Sanger sequencing in both the proband and the asymptomatic father (Fig. 2a,c). We then confirmed that the proband had compound heterozygosity, with the mutations in a trans configuration.

Patient 2 was a 48-year-old woman presented with progressive shuffling gait and decreased right arm swing since age of 30 years. Right-sided rigidity and bradykinesia were noted later. PD was diagnosed, and she responded well to levodopa. She had prominent psychiatric symptoms, including anxiety, depression and paranoid delusions, which symptoms occurred soon after the onset of right-side limbs slowness. Brain MRIs did not show structural lesions. A Tc-99 m TRODAT SPECT study showed reduced dopamine transporter activity in bilateral basal ganglia, with a more severe reduction on the left side. Her parents were cousins and had a consanguineous marriage. A genetic analysis with a targeted NGS panel, covering 40 genes associated with parkinsonism (supplementary Table 1), identified a homozygous c.991G > T (p.D331Y) mutation in PLA2G6 [5].

Patient 3 was a 34-year-old woman that started to complain of right-leg clumsiness at the age of 26 years. Right-arm clumsiness and gait disturbances followed 2 years later. Levodopa and dopamine agonists were given, with good motor responses; however, motor fluctuations and levodopa-induced dyskinesia developed 5 years after starting the medications. Depression and anxiety were also noted. Brain MRIs showed no structural change and the MMSE score was 28 out of 30 while she was 36 years old. A genetic analysis with a targeted NGS panel identified a homozygous c.991G > T (p.D331Y) mutation in PLA2G6. The clinical details were described previously [5]. This study was approved by the Institutional Review Board of National Taiwan University Hospital. Written informed consent was obtained from the participants.

\section{Systematic review of previous reports of patients with PLAN}

We searched the PubMed database for all English literature that had the terms: "PLA2G6 mutation" or "phospholipase A2 group VI gene mutation". We 
selected case reports or case series of patients with genetically confirmed PLAN, published in 2008-2019. Patients with childhood onset were excluded. We selected patients with adult-onset PLAN (onset age older than 18 years) and affected family members within the same family. However, family members with onset age less than 18 years were excluded. We summarized the clinical, genetic, and imaging characteristics of different genotypes to elucidate adult-onset PLA2G6.

We identified 32 patients from 23 articles that described patients with adult-onset PLAN. We did not enroll patients with onset age less than 18 years old. Including our three patients, we analyzed data for 35 patients. The clinical characteristics and genetic findings are summarized in Table 1 [1$3,6-32]$. The mean age of onset was $26.3 \pm 8.6$ years. The mean age of examination was $30.4 \pm 5.5$ years. At the initial presentation, 10 patients (28.6\%) had neuropsychiatric symptoms, rather than motor dysfunction. These symptoms included cognitive decline $(n=2)$ and depression with or without anxiety $(n=8)$ (Table 1$)$. The motor phenotypes were heterogeneous. Fourteen patients $(40.0 \%)$ had dystoniaparkinsonism, 17 patients (48.6\%) had levodopa-responsive, early-onset parkinsonism (EOPD), 3 patients (8.6\%) had hereditary spastic hemiparesis (HSP), and 1 patient (2.9\%) had cerebellar ataxia without signs of parkinsonism. The age at onset and gender distribution in patients with different clinical sub-groups were comparable (Table 2). The percentage of combined dystonia was higher in the group with dystoniaparkinsonism than in other groups $(P<0.001$; Table 2$)$. Of the 35 patients during examination, the main neurological signs included parkinsonism in 24 patients (68.6\%), neuropsychiatric symptoms in 19 patients (54.3\%), pyramidal signs with increased deep tendon reflexes in 19 patients (54.3\%), dystonia in 12 patients (34.3\%) and ataxia in 6 patient $\mathrm{s}$ (17.1\%) (Table 1). Brain MRIs were performed in all patients. Structural abnormalities were observed in 26 patients (74.3\%), including cortical atrophy ( $n=18,51.4 \%$ of all 35 patients), cerebellar atrophy ( $n=5,14.3 \%$ of all 35 patients), and hypointensity or suspected iron accumulations in the globus pallidus (observed in T2-weighted MRIs; $n=3,8.6 \%$ of all 35 patients). The percentage of cerebellar atrophy detected on brain MRIs was lower in patients with EOPD than in those with dystonia-parkinsonism, HSP, or ataxia $(P=$ 0.03 , Table 2). Tc-99 $\mathrm{m}$ TRODAT SPECT scans were performed in 12 patients, and all showed impaired and asymmetrical dopamine transporter activity. Six patients underwent brain PET studies, and all revealed hypometabolism in the cortical areas.

\section{Comparison of patients with different PLAN-related genotypes}

We next assigned patients to one of four groups, according to genotype, to reappraise potential genotypephenotype correlations (Table 3). All three of our patients had either homozygous or compound heterozygous p.D331Y mutations. Therefore, we compared the following four genotype groups: homozygous p.D331Y mutations $(n=5)$; compound heterozygous p.D331Y mutations with other variants $(\mathrm{n}=5)$; homozygous mutations other than p.D331Y $(n=17)$; and compound heterozygous mutations that did not include p.D331Y $(n=8)$ (Table 3). Of note, we found that the p.D331Y mutation in either the homozygous or compound heterozygous state, was more prevalent among Chinese and East Asians than among other ethnic groups $(P<0.001$ for homozygous p.D331Y mutations; $P=0.04$ for compound heterozygous p.D331Y with other variants, Table 3). Specifically, all patients with homozygous p.D331Y mutations and more than $85 \%$ of patients with compound heterozygous p.D331Y mutations were of Chinese ethnicity, which suggested a potential common founder effect. On the other hand, homozygous mutations other than p.D331Y were more prevalent among South Asians and Middle Eastern individuals, including Pakistanis, Saudi Arabians, Iranians, and Turks $(P<0.001$, Table 3$)$.

Phenotypically, all patients with homozygous p.D331Y mutations $(n=5)$ had EOPD, with a later age of onset (mean age of onset $33.0 \pm 4.8$ years), compared to patients with other genotypes $(P=0.03$, Table 3$)$. All five of these patients initially had an asymmetric onset of parkinsonism symptoms and responded well to levodopa. However, three of these five patients reported levodopa-induced dyskinesia. None exhibited dystonia, ataxia, an oculomotor abnormality, or autonomic dysfunction. Of note, the MRI findings of all five patients with homozygous p.D331Y showed no obvious structural changes, including T2-hypointensity in the globus pallidus. On the other hand, patients with mutations other than homozygous p.D331Y had an earlier age of onset $(P=0.03$, Table 3$)$ and often presented with dystoniaparkinsonism, rather than early-onset levodoparesponsive parkinsonism (30.7-57.1\%, Table 3). All carriers of mutations other than homozygous p.D331Y had abnormal brain MRI findings, including cortical and cerebellar atrophy, but without T2-hypointensity in the globus pallidus or iron accumulation.

Among 34 patients having mutations other than p.D331Y, nine patients carried homozygous c.2222G > A (p.R741Q) mutations and four patients had homozygous c.2239C > T (p.R747W) mutations. Of those 9 patients having homozygous p.R741Q mutations, six $(66.7 \%)$ had neuropsychiatric symptoms as the initial presentation and five $(55.6 \%)$ had features of dystonia-parkinsonism. Furthermore, of those 4 patients with homozygous p.R747W mutations, two had HSP phenotypes without signs of parkinsonism, one had dystonia-parkinsonism, and another one was clinically diagnosed with levodoparesponsive EOPD. 
Table 1 Clinical, genetic, and imaging characteristics of patients with genetically confirmed adult-onset PLAN in the literature

\begin{tabular}{|c|c|c|c|c|c|c|c|c|c|c|c|c|c|}
\hline \multirow{2}{*}{$\begin{array}{l}\text { Author, } \\
\text { year }\end{array}$} & \multirow[t]{2}{*}{ Patient } & \multirow[t]{2}{*}{ Ethnicity } & \multirow[t]{2}{*}{ Genotype } & \multirow[t]{2}{*}{ Type } & \multirow[t]{2}{*}{ Sex } & \multirow[t]{2}{*}{$\mathrm{AAO}$} & \multirow{2}{*}{$\begin{array}{l}\text { Initial } \\
\text { symptoms }\end{array}$} & \multicolumn{5}{|c|}{ Main symptoms during examination } & \multirow[t]{2}{*}{ MRI } \\
\hline & & & & & & & & EPS & Dystonia & $\begin{array}{l}\text { Pyramidal } \\
\text { signs }\end{array}$ & Ataxia & Psychiatric & \\
\hline \multicolumn{14}{|c|}{ Homozygous p.D331Y mutations $(\mathrm{N}=5)$} \\
\hline $\begin{array}{l}\text { Shi et al. } \\
2011[6]\end{array}$ & P1 & Chinese & $\begin{array}{l}\text { Homo c.991G > T (p. } \\
\text { D331Y) }\end{array}$ & EOPD & M & 37 & $\begin{array}{l}\text { Gait } \\
\text { disturbance }\end{array}$ & + & - & - & - & - & $\mathrm{nl}$ \\
\hline \multirow[t]{2}{*}{$\begin{array}{l}\text { Xie et al. } \\
2015[7]\end{array}$} & PA & Chinese & $\begin{array}{l}\text { Homo c.991G > T ( } p . \\
\text { D331Y) }\end{array}$ & EOPD & M & 36 & $\begin{array}{l}\text { Gait } \\
\text { disturbance }\end{array}$ & + & - & - & - & - & $\mathrm{nl}$ \\
\hline & PB & Chinese & $\begin{array}{l}\text { Homo c.991G > T (p. } \\
\text { D331Y) }\end{array}$ & EOPD & M & 36 & $\begin{array}{l}\text { Right hand } \\
\text { tremor }\end{array}$ & + & - & - & - & - & $\mathrm{nl}$ \\
\hline \multirow[t]{2}{*}{ This study } & P2 & Chinese & $\begin{array}{l}\text { Homo c.991G > T (p. } \\
\text { D331Y) }\end{array}$ & EOPD & $\mathrm{F}$ & 30 & Slowing gait & + & - & - & - & $D, P, A$ & $\mathrm{nl}$ \\
\hline & P3 & Chinese & $\begin{array}{l}\text { Homo c.991G > T ( } p . \\
\text { D331Y) }\end{array}$ & EOPD & $\mathrm{F}$ & 26 & $\begin{array}{l}\text { Right leg } \\
\text { clumsiness }\end{array}$ & + & - & + & - & $D, A$ & $\mathrm{nl}$ \\
\hline \multicolumn{14}{|c|}{ Compound heterozygous p.D331Y/ other mutations $(N=5)$} \\
\hline $\begin{array}{l}\text { Lu et al. } \\
2012[8]\end{array}$ & P3 & Chinese & $\begin{array}{l}\text { Compound hetero } \\
\text { c.991G > T (p. D331Y)/ } \\
\text { c.1077G > A (M358lfs X) }\end{array}$ & DP & $\mathrm{F}$ & 19 & $\begin{array}{l}\text { Unsteadiness } \\
\text { and } \\
\text { bradykinesia }\end{array}$ & + & + & - & + & $P, C$ & $\begin{array}{l}\text { CoA, } \\
\text { CeA }\end{array}$ \\
\hline \multirow[t]{2}{*}{$\begin{array}{l}\text { Chen } \\
\text { et al. } 2018 \\
\text { [9] }\end{array}$} & P3 & Chinese & $\begin{array}{l}\text { Compound hetero } \\
\text { c.991G > T(p.D331Y)/ } \\
\text { c.1982C > T }\end{array}$ & DP & M & 29 & $\begin{array}{l}\text { Walking } \\
\text { difficulty }\end{array}$ & + & - & - & + & - & CeA \\
\hline & P4 & Chinese & $\begin{array}{l}\text { Compound hetero } \\
\text { c.991G > T(p.D331Y)/ } \\
\text { c.2218G > A (p.G740R) }\end{array}$ & HSP & $\mathrm{F}$ & 31 & $\begin{array}{l}\text { Gait } \\
\text { disturbance }\end{array}$ & - & - & + & + & - & $\mathrm{CeA}$ \\
\hline $\begin{array}{l}\text { Ji et al. } \\
2019 \text { [10] }\end{array}$ & P1 & Chinese & $\begin{array}{l}\text { Compound hetero } \\
\text { c.991G > T (D331Y)/ } \\
\text { c.1648delC }\end{array}$ & Ataxia & $\mathrm{F}$ & 30 & Imbalance & - & - & + & + & $D$ & CeA \\
\hline This study & P1 & Chinese & $\begin{array}{l}\text { Compound hetero } \\
\text { c.991G > T (p. D331Y)/ } \\
\text { c.1077G > A (M358lfs X) }\end{array}$ & DP & M & 18 & $\begin{array}{l}\text { Depression } \\
\text { and } \\
\text { psychosis }\end{array}$ & + & + & - & - & $D, P, C$ & $\begin{array}{l}\text { CoA, } \\
\text { CeA }\end{array}$ \\
\hline
\end{tabular}

Homozygous Mutations other than p.D331Y $(N=17)$

\begin{tabular}{|c|c|c|c|c|c|c|c|c|c|c|c|c|c|}
\hline \multirow{2}{*}{$\begin{array}{l}\text { Paisa'n- } \\
\text { Ruiz } \\
\text { et al., } \\
2008 \text { [3] }\end{array}$} & $\begin{array}{l}\text { P1 of } \\
\text { F1 }\end{array}$ & Indian & $\begin{array}{l}\text { Homo c.2222G > A } \\
\text { (p.R741Q) }\end{array}$ & $\mathrm{DP}$ & $\mathrm{F}$ & 26 & $\begin{array}{l}\text { Cognitive } \\
\text { decline }\end{array}$ & + & + & + & - & $D, C$ & $\mathrm{CoA}$ \\
\hline & $\begin{array}{l}\text { P1 of } \\
\text { F2 }\end{array}$ & Pakistani & $\begin{array}{l}\text { Homo c. } 2239 \mathrm{C}>\mathrm{T} \\
\text { (p.R747W) }\end{array}$ & DP & $\mathrm{F}$ & 18 & Foot drag & + & + & + & - & C & $\mathrm{CoA}$ \\
\hline \multirow[t]{3}{*}{$\begin{array}{l}\text { Sina et al. } \\
2009 \text { [11] }\end{array}$} & P1 & Iranian & $\begin{array}{l}\text { Homo c.C1894T } \\
\text { (p.R632W) }\end{array}$ & $\mathrm{DP}$ & M & 25 & Foot drag & + & + & + & - & C & $\mathrm{CoA}$ \\
\hline & P2 & Iranian & $\begin{array}{l}\text { Homo c.C1894T } \\
\text { (p.R632W) }\end{array}$ & DP & M & 22 & Foot drag & + & + & + & - & C & $\mathrm{CoA}$ \\
\hline & P3 & Iranian & $\begin{array}{l}\text { Homo c.C1894T } \\
\text { (p.R632W) }\end{array}$ & $\mathrm{DP}$ & $\mathrm{F}$ & 21 & Foot drag & + & + & + & + & C & $\mathrm{CoA}$ \\
\hline $\begin{array}{l}\text { Agarwal } \\
\text { et al., } \\
2012 \text { [12] }\end{array}$ & P1 & Scandinavian & $\begin{array}{l}\text { Homo c.G238A } \\
\text { (p.A80T) }\end{array}$ & EOPD & $\mathrm{F}$ & 22 & Depression & + & + & + & - & $D, A, C$ & 1 \\
\hline \multirow[t]{2}{*}{$\begin{array}{l}\text { Virmani } \\
\text { et al., } \\
2014 \text { [13] }\end{array}$} & P1 & Indian & $\begin{array}{l}\text { Homo c.2222G > A } \\
\text { (p.R741Q) }\end{array}$ & $\mathrm{DP}$ & $\mathrm{F}$ & 25 & $\begin{array}{l}\text { Depression } \\
\text { and } \\
\text { psychosis }\end{array}$ & + & + & + & - & $D, P$ & $\mathrm{CoA}$ \\
\hline & P2 & Indian & $\begin{array}{l}\text { Homo c.2222G > A } \\
\text { (p.R741Q) }\end{array}$ & $\mathrm{DP}$ & $\mathrm{F}$ & 22 & Depression & + & + & + & - & - & $\mathrm{CoA}$ \\
\hline $\begin{array}{l}\text { Malaguti } \\
\text { et al. } 2015 \\
\text { [14] }\end{array}$ & P & Italian & $\begin{array}{l}\text { Homo c.C1547T } \\
\text { (p.A516W) }\end{array}$ & $\mathrm{DP}$ & $\mathrm{F}$ & 27 & $\begin{array}{l}\text { Stiff leg } \\
\text { sensation }\end{array}$ & + & + & + & - & C & I \\
\hline $\begin{array}{l}\text { Giri et al. } \\
2016 \text { [15] }\end{array}$ & P & Turkish & $\begin{array}{l}\text { Homo c. } 2239 C>T \\
\text { (p.R747W) }\end{array}$ & EOPD & $\mathrm{F}$ & 27 & $\begin{array}{l}\text { Left limb } \\
\text { slowness }\end{array}$ & + & - & - & - & $D, P, C$ & $\mathrm{CoA}$ \\
\hline
\end{tabular}


Table 1 Clinical, genetic, and imaging characteristics of patients with genetically confirmed adult-onset PLAN in the literature (Continued)

\begin{tabular}{|c|c|c|c|c|c|c|c|c|c|c|c|c|c|}
\hline \multirow{2}{*}{$\begin{array}{l}\text { Author, } \\
\text { year }\end{array}$} & \multirow[t]{2}{*}{ Patient } & \multirow[t]{2}{*}{ Ethnicity } & \multirow[t]{2}{*}{ Genotype } & \multirow[t]{2}{*}{ Type } & \multirow[t]{2}{*}{ Sex } & \multirow[t]{2}{*}{$\mathrm{AAO}$} & \multirow{2}{*}{$\begin{array}{l}\text { Initial } \\
\text { symptoms }\end{array}$} & \multicolumn{5}{|c|}{ Main symptoms during examination } & \multirow[t]{2}{*}{ MRI } \\
\hline & & & & & & & & EPS & Dystonia & $\begin{array}{l}\text { Pyramidal } \\
\text { signs }\end{array}$ & Ataxia & Psychiatric & \\
\hline $\begin{array}{l}\text { Ozes et al. } \\
2017 \text { [16] }\end{array}$ & P2 & Turkish & $\begin{array}{l}\text { Homo c.2239C > T } \\
\text { (p.R747W) }\end{array}$ & HSP & M & 21 & $\begin{array}{l}\text { Scissoring } \\
\text { gait }\end{array}$ & - & - & + & + & - & 1 \\
\hline $\begin{array}{l}\text { Koh et al. } \\
2018[17]\end{array}$ & D II-3 & Japanese & $\begin{array}{l}\text { Homo c.1904G > A } \\
\text { (p.R635Q) }\end{array}$ & $\mathrm{HSP}$ & $\mathrm{F}$ & 66 & $\begin{array}{l}\text { Gait } \\
\text { impairment }\end{array}$ & + & - & + & - & - & $\mathrm{nl}$ \\
\hline \multirow{4}{*}{$\begin{array}{l}\text { Bohlega } \\
\text { et al. } 2018 \\
{[18]}\end{array}$} & $\begin{array}{l}\text { P1 of } \\
\text { F1 }\end{array}$ & $\begin{array}{l}\text { Saudi } \\
\text { Arabian }\end{array}$ & $\begin{array}{l}\text { Homo c.2222G > A } \\
\text { (p.R741Q) }\end{array}$ & EOPD & $\mathrm{F}$ & 26 & $\begin{array}{l}\text { Depression, } \\
\text { bradykinesia }\end{array}$ & + & - & + & - & $D, C$ & $\mathrm{COA}$ \\
\hline & $\begin{array}{l}\text { P2 of } \\
\text { F1 }\end{array}$ & $\begin{array}{l}\text { Saudi } \\
\text { Arabian }\end{array}$ & $\begin{array}{l}\text { Homo c.2222G > A } \\
\text { (p.R741Q) }\end{array}$ & EOPD & M & 22 & $\begin{array}{l}\text { Depression, } \\
\text { tremor }\end{array}$ & + & - & + & - & $D, C$ & $\mathrm{COA}$ \\
\hline & $\begin{array}{l}\text { P3 of } \\
\text { F1 }\end{array}$ & $\begin{array}{l}\text { Saudi } \\
\text { Arabian }\end{array}$ & $\begin{array}{l}\text { Homo c.2222G > A } \\
\text { (p.R741Q) }\end{array}$ & EOPD & $\mathrm{F}$ & 23 & Bradykinesia & + & - & + & - & C & $\mathrm{COA}$ \\
\hline & $\begin{array}{l}\text { P1 of } \\
\text { F2 }\end{array}$ & $\begin{array}{l}\text { Saudi } \\
\text { Arabian }\end{array}$ & $\begin{array}{l}\text { Homo c.2222G > A } \\
\text { (p.R741Q) }\end{array}$ & EOPD & M & 25 & $\begin{array}{l}\text { Cognitive } \\
\text { decline }\end{array}$ & + & - & + & - & C & $\mathrm{COA}$ \\
\hline $\begin{array}{l}\text { Rohani } \\
\text { et al. } 2018 \\
\text { [19] }\end{array}$ & P & $\begin{array}{l}\text { Saudi } \\
\text { Arabian }\end{array}$ & $\begin{array}{l}\text { Homo p. } \\
\text { Ala681Cysfs*92 }\end{array}$ & $\mathrm{DP}$ & M & 18 & $\begin{array}{l}\text { Bradykinesia, } \\
\text { tremor }\end{array}$ & + & + & + & - & $\mathrm{P}$ & $\mathrm{nl}$ \\
\hline \multicolumn{14}{|c|}{ Compound Heterozygous mutations other than p.D331Y $(\mathrm{N}=8)$} \\
\hline \multirow[t]{3}{*}{$\begin{array}{l}\text { Yoshino } \\
\text { et al. } 2010 \\
{[20]}\end{array}$} & PA & Japanese & $\begin{array}{l}\text { Compound hetero } \\
\text { c.C216A (p. p.F72L) } \\
\text { /c.G1904A (p.R635Q) }\end{array}$ & EOPD & $\mathrm{F}$ & 20 & $\begin{array}{l}\text { Resting } \\
\text { tremor, } \\
\text { unsteady gait }\end{array}$ & + & - & - & - & $D, P, C$ & $\begin{array}{l}\mathrm{COA}, \\
\mathrm{I}\end{array}$ \\
\hline & PB1 & Japanese & $\begin{array}{l}\text { Compound hetero } \\
\text { c.C1354T (p.Q452X) } \\
\text { /c.G1904A (p.R635Q) }\end{array}$ & EOPD & M & 25 & $\begin{array}{l}\text { Bradykinesia, } \\
\text { gait } \\
\text { disturbance }\end{array}$ & + & - & - & - & C & $\mathrm{COA}$ \\
\hline & PB2 & Japanese & $\begin{array}{l}\text { Compound hetero } \\
\text { c.C1354T (p.Q452X) } \\
\text { /c.G1904A (p.R635Q) }\end{array}$ & EOPD & M & 30 & $\begin{array}{l}\text { Bradykinesia, } \\
\text { gait } \\
\text { disturbance }\end{array}$ & + & - & - & - & $P, C$ & $\mathrm{COA}$ \\
\hline $\begin{array}{l}\text { Bower } \\
\text { et al., } \\
2011 \text { [21] }\end{array}$ & P1 & European & $\begin{array}{l}\text { Compound hetero } \\
\text { c.C4A (p.Q2K)/Del Ex } 3 \\
\text { (pL71_S142del) }\end{array}$ & $\mathrm{DP}$ & $\mathrm{F}$ & 18 & Depression & + & + & + & - & $\mathrm{D}$ & GP \\
\hline $\begin{array}{l}\text { Kim et al. } \\
2015 \text { [22] }\end{array}$ & $\mathrm{P} 1$ & Korean & $\begin{array}{l}\text { Compound hetero } \\
\text { c.G1039A (p.G347R) } \\
\text { /c.C1670T (p.S557L) }\end{array}$ & DP & $\mathrm{F}$ & 22 & $\begin{array}{l}\text { Unsteady gait } \\
\text { and falls }\end{array}$ & + & + & + & + & C & $\begin{array}{l}\mathrm{CeA}, \\
\mathrm{l}\end{array}$ \\
\hline \multirow[t]{2}{*}{$\begin{array}{l}\text { Wirth } \\
\text { et al. } 2017 \\
\text { [23] }\end{array}$} & P1 & Caucasian & $\begin{array}{l}\text { Compound hetero } \\
\text { c.109C > T (p.R37X)/ } \\
\text { c.2321G > T } \\
\text { (p.Ser774lso) }\end{array}$ & EOPD & M & 23 & $\begin{array}{l}\text { Depression } \\
\text { and anxiety }\end{array}$ & + & + & - & - & $D, P, A$ & $\mathrm{nl}$ \\
\hline & P2 & Caucasian & $\begin{array}{l}\text { Compound hetero } \\
\text { c.758G > T (Gly253Val)/ } \\
\text { c.2341G > A (Ala781Thr) }\end{array}$ & EOPD & M & 27 & $\begin{array}{l}\text { Left leg } \\
\text { tremor and } \\
\text { anxiety }\end{array}$ & + & - & + & - & $P, A, C$ & $\mathrm{nl}$ \\
\hline $\begin{array}{l}\text { Chen } \\
\text { et al.2018 } \\
\text { [9] }\end{array}$ & P2 & Chinese & $\begin{array}{l}\text { Compound } \\
\text { heteroc.668C > T } \\
\text { (p.P223L) /c.1915G > } \\
\text { A(p.A639T) }\end{array}$ & EOPD & M & 27 & $\begin{array}{l}\text { Walking } \\
\text { difficulty }\end{array}$ & + & - & + & - & - & $\begin{array}{l}\mathrm{CeA}, \\
\mathrm{GP}, \mathrm{I}\end{array}$ \\
\hline
\end{tabular}

Abbreviations: +, present; -, absent or not reported; $F 1$ family 1; $P 1$ patient 1; DP dystonia-parkinsonism; EOPD early-onset Parkinson's disease; HSP hereditary spastic hemiplegia; $A A O$ age at onset; $E P S$ extrapyramidal sign; $M R I$ magnetic resonance imaging; $D$ depression; $P$ psychosis; $A$ anxiety; $C$ cognitive decline; CoA Cortical atrophy; CeA Cerebellar atrophy; GP T2- hypointensity in globus pallidus; I Iron accumulation; $n$ / normal; hypo hypometabolism; NA not available

\section{Discussion and conclusions}

We described the clinical, genetic, and neuroimaging aspects of three patients with adult-onset PLAN. One patient had compound heterozygous p.D331Y and M358IfsX frame-shift mutations and presented with dystonia-parkinsonism. The other two patients had homozygous p.D331Y mutations and presented with levodopa-responsive early-onset parkinsonism.
After identifying more than 20 PLA2G6 mutations in previous reports [1-3, 6-32], we observed that the c.991G $>\mathrm{T}$ (p. D331Y) mutation in PLA2G6 was almost exclusively found in Chinese patients, suggesting a common founder effect of this variant in Chinese populations. Moreover, patients with homozygous c.991G > T (p.D331Y) mutations showed purely early-onset parkinsonism features with good levodopa 
Table 2 Comparison of patients with different clinical subtypes of PLAN

\begin{tabular}{|c|c|c|c|c|c|}
\hline Characteristics & $\begin{array}{l}\text { Dystonia-Parkinsonism } \\
N=14\end{array}$ & $\begin{array}{l}\text { Early-onset PD } \\
N=17\end{array}$ & $\begin{array}{l}\mathrm{HSP} \\
N=3\end{array}$ & $\begin{array}{l}\text { Ataxia } \\
N=1\end{array}$ & $P$-value \\
\hline Age at onset, years & $21.7 \pm 3.9$ & $27.2 \pm 5.1$ & $46.0 \pm 23.5$ & 30 & 0.10 \\
\hline Age at examination, years & $32.5 \pm 4.9$ & $33.7 \pm 8.1$ & N.A. & 31 & 0.26 \\
\hline Sex, male & $5(35.7 \%)$ & $10(58.8 \%)$ & $1(33.3 \%)$ & 0 & 0.38 \\
\hline \multicolumn{6}{|l|}{ Main symptoms and signs } \\
\hline Parkinsonism & $14(100.0 \%)$ & $17(100.0 \%)$ & 0 & 0 & 1.00 \\
\hline Dystonia & $13(92.8 \%)$ & $2(11.8 \%)$ & 0 & 0 & $<0.001^{* *}$ \\
\hline Pyramidal sign & $11(78.6 \%)$ & $8(47.1 \%)$ & $3(100 \%)$ & 1 & 0.32 \\
\hline Cognitive decline & $9(64.3 \%)$ & $10(58.8 \%)$ & $1(33.3 \%)$ & 0 & 0.91 \\
\hline Depression/Anxiety & $4(28.6 \%)$ & $7(41.2 \%)$ & $1(33.3 \%)$ & 1 & 0.78 \\
\hline Psychosis & $4(28.6 \%)$ & $6(35.3 \%)$ & 0 & 0 & 0.93 \\
\hline \multicolumn{6}{|l|}{ Brain MRI findings } \\
\hline Cortical atrophy & $9(64.3 \%)$ & $8(47.1 \%)$ & 0 & 0 & \\
\hline Cerebellar atrophy & $3(21.4 \%)$ & $1(5.9 \%)$ & $1(33.3 \%)$ & 1 & $0.03^{*}$ \\
\hline Hypo-intensity in GP & $1(7.1 \%)$ & $1(5.9 \%)$ & 0 & 0 & 0.98 \\
\hline
\end{tabular}

Data are the number (\%) or the mean \pm SD. PLAN, Phospholipase A2 group Vl-associated neurodegeneration; PD, Parkinson's disease; HSP, hereditary spastic paraparesis; MRI, magnetic resonance imaging; GP, globus pallidus. ${ }^{* *} P<0.05$; ${ }^{* *} P<0.01$. $P$-values that compare individual characteristics between groups with dystonia-parkinsonism and early-onset PD were evaluated with an analysis of variance. Variables without a normal distribution were compared with the KruskalWallis test, the non-parametric equivalent of the independent sample t-test

responses. In contrast, patients with other genotypes, including compound heterozygous c.991G > T (p.D331Y) mutations and other variants, predominantly presented with dystoniaparkinsonism and HSP. Our results further support previous studies that PLA2G6 mutations had both clinical and genetic heterogeneity. Moreover, our findings suggested that the c.991G > T (p.D331Y) mutation was a potentially common founder mutation in populations of Chinese ethnicity.

The homozygous c.991G > T (p.D331Y) mutation in PLA2G6 was first identified in patients diagnosed with

Table 3 Comparison of patients with different PLAN-related genotypes

\begin{tabular}{|c|c|c|c|c|c|}
\hline Characteristics & $\begin{array}{l}\text { Homozygous p.D331Y } \\
\text { mutations } \\
N=5\end{array}$ & $\begin{array}{l}\text { Compound heterozygous p.D331Y/ } \\
\text { other mutations } \\
N=5\end{array}$ & $\begin{array}{l}\text { Homozygous } \\
\text { Mutations other than } \\
\text { p.D331Y } \\
\mathrm{N}=17\end{array}$ & $\begin{array}{l}\text { Compound } \\
\text { Heterozygous } \\
\text { mutations other than } \\
\text { p.D331Y } \\
N=8\end{array}$ & $\begin{array}{l}P \text { - } \\
\text { value }\end{array}$ \\
\hline Age at onset, years & $33.0 \pm 4.8$ & $25.4 \pm 6.3$ & $23.2 \pm 11.0$ & $16.8 \pm 9.9$ & 0.23 \\
\hline Sex, male & $2(50.0 \%)$ & $2(40.0 \%)$ & $6(35.3 \%)$ & $5(62.5 \%)$ & 0.41 \\
\hline \multicolumn{6}{|l|}{ Ethnicity } \\
\hline Chinese & $5(100.0 \%)$ & $5(100.0 \%)$ & 0 & $1(12.5 \%)$ & $\begin{array}{l}< \\
0.001^{* *}\end{array}$ \\
\hline East Asian & 0 & 0 & $1(4.8 \%)$ & $4(50.0 \%)$ & $0.01^{*}$ \\
\hline South Asian & 0 & 0 & $4(19.0 \%)$ & 0 & 0.16 \\
\hline Middle Eastern & 0 & 0 & $14(66.7 \%)$ & 0 & $\begin{array}{l}< \\
0.01^{* *}\end{array}$ \\
\hline Caucasian & 0 & 0 & $2(9.5 \%)$ & $3(37.5 \%)$ & 0.11 \\
\hline \multicolumn{6}{|c|}{ Main clinical subtypes } \\
\hline $\begin{array}{l}\text { Dystonia- } \\
\text { parkinsonism }\end{array}$ & 0 & $3(60.0 \%)$ & 9 (52.9\%) & $2(25.0 \%)$ & 0.07 \\
\hline Early-onset PD & $5(100.0 \%)$ & 0 & $6(35.3 \%)$ & $6(75.0 \%)$ & $0.01^{*}$ \\
\hline HSP & 0 & $1(20.0 \%)$ & $2(11.8 \%)$ & 0 & 0.78 \\
\hline Ataxia & 0 & $1(20.0 \%)$ & 0 & 0 & 0.68 \\
\hline
\end{tabular}

Data are the number (\%) or the mean \pm SD. PLAN, Phospholipase A2 group VI-associated neurodegeneration; PD, Parkinson's disease; HSP, hereditary spastic paraparesis. ${ }^{* *} P<0.05 ;{ }^{* *} P<0.01$. $P$-values that compare individual characteristics between four groups with different genotypes were evaluated with an analysis of variance. Variables without a normal distribution were compared with the Kruskal-Wallis test, the non-parametric equivalent of the independent sample t-test 
levodopa-responsive EOPD $(25,30)$. Consistent with initial reports, our two patients with this same mutation had comparable phenotypes and there were no signs of iron accumulation in the globus pallidus in brain MRIs. The PLA2G6 gene encodes a group of VIA calciumindependent phospholipase A2 (iPLA2 $\beta$ ) enzymes, which participate in various cellular functions, including phospholipid metabolism, membrane homeostasis, calcium signaling, apoptosis, and mitochondrial function. Thus, these functions could be perturbed by PLA2G6 gene mutations [24]. A homozygous c.991G $>\mathrm{T}$ (p.D331Y) knock-in mouse model exhibited dopaminergic neuron degeneration in the substantia nigra, caused by mitochondrial dysfunction, elevated endoplasmic reticulum stress, and transcriptional abnormalities, conforming its pathogenicity in neuronal degeneration [24]. A previous in vitro study showed that patients with the homozygous c.991G > T (p.D331Y) mutation had 30\% enzymatic activity remaining in the PLA2G6 protein by using the modified kit originally designed for cytosolic $\mathrm{Ca}^{+}$-dependent PLA2 (cPLA2) (cPLA2 Assay Kit, Cayman Chemicals) in HEK293 T cells transfected with human mutant constructs (25). An incomplete loss of the iPLA2 $\beta$ enzyme could partially explain the relatively milder clinical and neuroimaging phenotypes of patients with homozygous c.991G > T (p.D331Y) mutations, compared to patients with other mutations in PLA2G6.

The crystal structure of iPLA2 $\beta$ is complex, and the different mutation sites are disparately located on the enzyme [1]. Different mutation sites in the different domains of iPLA2 $\beta$ can lead to different changes in enzymatic activities; however, iPLA2 $\beta$ enzyme activity is the crucial factor that affects the clinical phenotypes of PLAN [33]. For example, the activity of iPLA2 $\beta$ was reduced by $70 \%$, with some remaining enzyme activity, in cells that expressed p.D331Y, compared to wild-type cells [6]; in contrast, iPLA2 $\beta$ with the p.H597fx69 frameshift mutation exhibited less than $6 \%$ enzyme activity compared to the wild-type iPLA2 $\beta$ [34]. The compound heterozygous genotype of p.D331Y and p.M358IfsX mutations was first reported in a Chinese patient that presented with dystonia-parkinsonism [8]. Our first index patient with these same mutations shared similar clinical manifestations, including early age at onset, prominent dystonia, depression, and cognitive decline. The MRI scans in both patients did not show signs of iron accumulations in globus pallidus. The c.1077G > A (p.M358IfsX) mutation, which is located at the splicing junction, resulted in c.1074_1077del.GTCG, and caused aberrant RNA splicing, which resulted in a frame-shift mutation. We hypothesized that this frame-shift mutation might perturb iPLA2 $\beta$ enzyme activity. Indeed, patients with homozygous p.D331Y mutations often presented with levodopa-responsive EOPD, but patients with compound heterozygous p.D331Y and p.M358IfsX mutations often presented with dystonia-parkinsonism, with features of dystonia, pyramidal signs, ataxia, and psychiatric comorbidities. Accordingly, we speculated that iPLA2 $\beta$ enzyme activity might be less in neurons that expressed the p.M358IfsX mutation than in neurons that expressed the p.D331Y mutation. Further functional studies are needed to clarify the molecular mechanism that leads to the clinical heterogeneity associated with different mutations in PLA2G6.

In addition to levodopa-responsive EOPD and dystoniaparkinsonism, 3 patients (from the literature review) having PLA2G6 mutations and displayed HSP, which findings extended our current understanding of the spectrum of clinical phenotypes associated with adult-onset PLAN. Moreover, one patient had compound heterozygous c.991G $>\mathrm{T}$ (D331Y) and c.1648delC mutations and presented with prominent ataxia, pyramidal signs, and depression, but did not show signs of parkinsonism or dystonia [10]. That presentation was also rare in adult-onset PLAN. In addition to diverse motor symptoms, neuropsychiatric symptoms comprise one of the main features of adult-onset PLAN. Our index patient with compound heterozygous p.D331Y and p.M358IfsX mutations had severe depression, and one of the two patients with a homozygous p.D331Y mutation also had depression and prominent psychotic symptoms. Many reports also found neuropsychiatric symptoms or behavioral changes in the initial presentations of patients with PLA2G6 mutations $[3,12,13,18,23,25,27]$. Early cognitive decline or psychiatric symptoms with motor symptoms, such as parkinsonism or dystonia, should prompt clinicians to include PLAN in their differential diagnoses. In those cases, genetic testing for PLA2G6 mutations are warranted, even when brain MRIs show no iron accumulation in the globus pallidus. Moreover, due to the susceptibility to developing psychotic symptoms, dopaminergic agents for treating parkinsonism symptoms in these patients should be carefully titrated and closely monitored to identify psychiatric complications during treatment.

This study had some limitations. First, due to the rarity of the disease, the number of cases was limited. This limitation might have attenuated the statistical power of the results in the comparison of clinical phenotypes among different PLA2G6 mutations. Moreover, due to the diverse clinical manifestations, including motor and neuropsychiatric involvements, the clinical assessments might not have been comprehensive, in every case, in the literature review. Furthermore, although our study focused on adult-onset PLAN, some family members of the index patient may have symptoms since the childhood and we still enrolled these patients into the analysis to have a better understanding of the clinical presentation of the same mutation within the family. Future studies should include thorough clinical, functional 
imaging, and genetic analyses, with a long-term followup, to provide a better understanding of the correlations between genotype and phenotype in PLAN.

In summary, our findings suggested that adult-onset PLAN could present with purely early-onset levodoparesponsive parkinsonism features, with no brain iron accumulation, particularly in patients with homozygous c.991G > T (p.D331Y) mutations. The homozygous c.991G > T (p.D331Y) mutations were exclusively found in patients of Chinese ethnicity, suggesting a common founder effect. Patients with compound heterozygous mutations had heterogeneous phenotypes, including dystonia-parkinsonism, HSP, and ataxia, and these were often combined with diverse neuropsychiatric symptoms. The wide intra- and inter-familial phenotypic variability of adult-onset PLAN may contribute from other environmental and/or genetic modifiers that might probably modulate the disease presentation. Future studies encompassing whole genome sequencing in patients with PLAN are needed to delineate the potential modifier genes of this disease.

\section{Supplementary information}

Supplementary information accompanies this paper at https://doi.org/10. 1186/s12883-020-01684-6.

Additional file 1 Supplementary Table 1. The 40 candidate genes involved in PD and related neurodegenerative disorders that were used for targeted NGS in the study.

\section{Abbreviations}

PLA2G6: Phospholipase A2 group VI; PLAN: Phospholipase A2 group VI (PLA2G6) mutations associated with neurodegeneration

\section{Acknowledgements}

We thank the patient in this case study and his family for participating in the study. We are also grateful to the staff of the Second Core Lab, Department of Medical Research, National Taiwan University Hospital, for technical support during the study.

\section{Authors' contributions}

Study concept and design: $\mathrm{CH}$. Acquisition of data: $\mathrm{YT}, \mathrm{HY}, \mathrm{PL}$ and $\mathrm{CH}$. Analysis and interpretation of data: $\mathrm{YT}$ and $\mathrm{CH}$. Drafting of the manuscript: YT. Critical revision of the manuscript for important intellectual content: $\mathrm{CH}$. Study supervision: $\mathrm{CH}$. All authors have read and approved the final version of the manuscript.

\section{Funding}

This work was supported by the National Taiwan University Hospital (UN108-023).

\section{Availability of data and materials}

All the relevant raw data in the current study will be freely available to any scientist wishing to use them for non-commercial purposes, without breaching participant confidentiality.

\section{Ethics approval and consent to participate}

This study was approved by the institutional review board of National Taiwan University Hospital and all study participants gave their inform consents before entering the study. Our study is in accordance with the Declaration of Helsinki and CARE guidelines/methodology. All authors agreed the Publish Statements of BMC Neurology.

\section{Consent for publication}

Written consent for publication was obtained from each of the participants in this study.

\section{Competing interests}

All authors have no competing interests or conflicts of interest

Chin-Hsien Lin is a member of the editorial board (Associate Editor) of this journal.

\section{Author details}

'Department of Neurology, National Taiwan University Hospital, No. 7, Chung-Shan South Road, Taipei 100, Taiwan. ${ }^{2}$ Graduate Institute of Medical Genomics and Proteomics, National Taiwan University College of Medicine, Taipei, Taiwan. ${ }^{3}$ Department of Medical Genetics, National Taiwan University Hospital, Taipei, Taiwan.

Received: 21 November 2019 Accepted: 11 March 2020

Published online: 17 March 2020

\section{References}

1. Guo YP, Tang BS, Guo JF. PLA2G6-associated Neurodegeneration (PLAN): review of clinical phenotypes and genotypes. Front Neurol. 2018;9:1100.

2. Darling A, Aguilera-Albesa S, Tello CA, Serrano M, Tomás M, Camino-León R, et al. PLA2G6-associated neurodegeneration: new insights into brain abnormalities and disease progression. Parkinsonism Relat Disord. 2019;61:179-86.

3. Paisan-Ruiz C, Bhatia KP, Li A, Hernandez D, Davis M, Wood NW, et al. Characterization of PLA2G6 as a locus for dystonia-parkinsonism. Ann Neurol. 2009:65:19-23.

4. Hayflick SJ, Kurian MA, Hogarth P. Neurodegeneration with brain iron accumulation. Handb Clin Neurol. 2018;147:293-305.

5. Fan SP, Lee NC, Lin CH. Clinical and electrophysiological characteristics of a type 1 sialidosis patient with a novel deletion mutation in NEU1 gene. J Formos Med Assoc. 2020;119:406-12.

6. Shi $\mathrm{CH}$, Tang BS, Wang L, Lv ZY, Wang J, Luo LZ, et al. PLA2G6 gene mutation in autosomal recessive early-onset parkinsonism in a Chinese cohort. Neurology. 2011;77:75-81.

7. Xie F, Cen Z, Ouyang Z, Wu S, Xiao J, Luo W. Homozygous p.D331Y mutation in PLA2G6 in two patients with pure autosomal-recessive earlyonset parkinsonism: further evidence of a fourth phenotype of PLA2G6associated neurodegeneration. Parkinsonism Relat Disord. 2015;21:420-2.

8. Lu CS, Lai SC, Wu RM, Weng YH, Huang CL, Chen RS, et al. PLA2G6 mutations in PARK14-linked young-onset parkinsonism and sporadic Parkinson's disease. Am J Med Genet B Neuropsychiatr Genet. 2012;159B:183-91.

9. Chen YJ, Chen YC, Dong HL, Li LX, Ni W, Li HF, et al. Novel PLA2G6 mutations and clinical heterogeneity in Chinese cases with phospholipase A2-associated neurodegeneration. Parkinsonism Relat Disord. 2018;49:88-94.

10. Ji Y, Li Y, Shi C, Gao Y, Yang J, Liang D, et al. Identification of a novel mutation in PLA2G6 gene and phenotypic heterogeneity analysis of PLA2G6-related neurodegeneration. Parkinsonism Relat Disord. 2019;65:159-64.

11. Sina F, Shojaee S, Elahi E, Paisan-Ruiz C. R632W mutation in PLA2G6 segregates with dystonia-parkinsonism in a consanguineous Iranian family. Eur J Neurol. 2009;16:101-4.

12. Agarwal P, Hogarth P, Hayflick S, MacLeod P, Kuriakose R, McKenzie J, et al. Imaging striatal dopaminergic function in phospholipase A2 group VIrelated parkinsonism. Mov Disord. 2012;27:1698-9.

13. Virmani T, Thenganatt MA, Goldman JS, Kubisch C, Greene PE, Alcalay RN Oculogyric crises induced by levodopa in PLA2G6 parkinsonism-dystonia. Parkinsonism Relat Disord. 2014:20:245-7.

14. Malaguti MC, Melzi V, Di Giacopo R, Monfrini E, Di Biase E, Franco G, et al. A novel homozygous PLA2G6 mutation causes dystonia-parkinsonism. Parkinsonism Relat Disord. 2015;21:337-9.

15. Giri A, Guven G, Hanagasi H, Hauser AK, Erginul-Unaltuna N, Bilgic B, et al. PLA2G6 Mutations Related to Distinct Phenotypes: A New Case with Earlyonset Parkinsonism. Tremor Other Hyperkinet Mov (N Y). 2016;6:363.

16. Ozes B, Karagoz N, Schule R, Rebelo A, Sobrido MJ, Harmuth F, et al. PLA2G6 mutations associated with a continuous clinical spectrum from neuroaxonal dystrophy to hereditary spastic paraplegia. Clin Genet. 2017;92:534-9.

17. Koh K, Ichinose $Y$, Ishiura H, Nan H, Mitsui J, Takahashi J, et al. PLA2G6associated neurodegeneration presenting as a complicated form of hereditary spastic paraplegia. J Hum Genet. 2019;64:55-9. 
18. Bohlega SA, Al-Mubarak BR, Alyemni EA, Abouelhoda M, Monies D, Mustafa AE, et al. Clinical heterogeneity of PLA2G6-related parkinsonism: analysis of two Saudi families. BMC Res Notes. 2016;9:295.

19. Rohani M, Shahidi G, Vali F, Lang AE, Slow E, Gahl WA, et al. Oculogyric crises in PLA2G6 associated neurodegeneration. Parkinsonism Relat Disord. 2018:52:111-2.

20. Yoshino H, Tomiyama H, Tachibana N, Ogaki K, Li Y, Funayama M, et al. Phenotypic spectrum of patients with PLA2G6 mutation and PARK14-linked parkinsonism. Neurology. 2010;75:1356-61.

21. Bower MA, Bushara K, Dempsey MA, Das S, Tuite PJ. Novel mutations in siblings with later-onset PLA2G6-associated neurodegeneration (PLAN). Mov Disord. 2011;26:1768-9.

22. Kim YJ, Lyoo CH, Hong S, Kim NY, Lee MS. Neuroimaging studies and whole exome sequencing of PLA2G6-associated neurodegeneration in a family with intrafamilial phenotypic heterogeneity. Parkinsonism Relat Disord. 2015;21:402-6.

23. Wirth T, Weibel S, Montaut S, Bigaut K, Rudolf G, Chelly J, et al. Severe earlyonset impulsive compulsive behavior and psychosis in PLA2G6-related juvenile Parkinson's disease. Parkinsonism Relat Disord. 2017;41:127-9.

24. Chiu CC, Lu CS, Weng YH, Chen YL, Huang YZ, Chen RS, et al. PARK14 (D331Y) PLA2G6 causes early-onset degeneration of Substantia Nigra dopaminergic neurons by inducing mitochondrial dysfunction, ER stress, Mitophagy impairment and transcriptional Dysregulation in a Knockin mouse model. Mol Neurobiol. 2019;56:3835-53.

25. Erro R, Balint B, Kurian MA, Brugger F, Picillo M, Barone P, et al. Early Ataxia and subsequent parkinsonism: PLA2G6 mutations cause a continuum rather than three discrete phenotypes. Mov Disord Clin Pract. 2017:4:125-8.

26. Ferini-Strambi L, Marelli S, Moccia M, Erro R, Ciacci C, Barone P. Malabsorption is uncommon in restless legs syndrome. Mov Disord. 2011; 26:1767-8.

27. Kamel WA, Al-Hashel JY, Abdulsalam AJ, Damier P, Al-Mejalhem AY. PLA2G6-related parkinsonism presenting as adolescent behavior. Acta Neurol Belg. 2019;119:621-2.

28. Karkheiran S, Shahidi GA, Walker RH, Paisan-Ruiz C. PLA2G6-associated Dystonia-Parkinsonism: Case Report and Literature Review. Tremor Other Hyperkinet Mov (N Y). 2015;5:317.

29. Marras C, Lang A, van de Warrenburg BP, Sue CM, Tabrizi SJ, Bertram L, et al. Nomenclature of genetic movement disorders: recommendations of the international Parkinson and movement disorder society task force. Mov Disord. 2016;31:436-57.

30. Michelis JP, Hattingen E, Gaertner FC, Minnerop M, Träber F, Biskup S, et al. Expanded phenotype and hippocampal involvement in a novel compound heterozygosity of adult PLA2G6 associated neurodegeneration (PARK14). Parkinsonism Relat Disord. 2017:37:111-3.

31. Tian JY, Tang BS, Shi CH, Lv ZY, Li K, Yu RL, et al. Analysis of PLA2G6 gene mutation in sporadic early-onset parkinsonism patients from Chinese population. Neurosci Lett. 2012;514:156-8.

32. Yamashita C, Funayama M, Li Y, Yoshino H, Yamada H, Seino $Y$, et al. Mutation screening of PLA2G6 in Japanese patients with early onset dystonia-parkinsonism. J Neural Transm (Vienna). 2017;124:431-5.

33. Engel LA, Jing Z, O'Brien DE, Sun M, Kotzbauer PT. Catalytic function of PLA2G6 is impaired by mutations associated with infantile neuroaxonal dystrophy but not dystonia-parkinsonism. PLoS One. 2010;5:e12897.

34. Gui YX, Xu ZP, Wen L, Liu HM, Zhao JJ, Hu XY. Four novel rare mutations of PLA2G6 in Chinese population with Parkinson's disease. Parkinsonism Relat Disord. 2013;19:21-6.

\section{Publisher's Note}

Springer Nature remains neutral with regard to jurisdictional claims in published maps and institutional affiliations.

Ready to submit your research? Choose BMC and benefit from:

- fast, convenient online submission

- thorough peer review by experienced researchers in your field

- rapid publication on acceptance

- support for research data, including large and complex data types

- gold Open Access which fosters wider collaboration and increased citations

- maximum visibility for your research: over $100 \mathrm{M}$ website views per year

At BMC, research is always in progress.

Learn more biomedcentral.com/submissions 